\title{
A DSM-BASED “2.0” SYSTEM FOR HUMAN INTERVENTION PLANNING AND SCHEDULING IN FACILITIES EMITTING IONIZING RADIATIONS
}

\author{
Mathieu Baudin ${ }^{1}$, Pierre Bonnal ${ }^{2}$ and Jurgen De Jonghe ${ }^{2}$ \\ ${ }^{1}$ CERN (European Organization for Nuclear Research), Geneva, Switzerland and \\ LCPI (Product Design and Innovation Laboratory) Arts \& Métiers ParisTech, France \\ ${ }^{2}$ CERN (European Organization for Nuclear Research), Geneva, Switzerland
}

\begin{abstract}
To efficiently and safely plan, schedule and control its interventions in underground facilities, which are subject to ionizing radiations, CERN is currently developing a collaborative Web-based system. A similar project for maintenance management is also under way. On top of presenting their key requirements, this paper shows how the implementation of DSM can enhance a so-called "Web 2.0" or collaborative dimension by bringing an intuitive and fair way of taking the dependencies between several activities into account. It is also discussed that the incoherencies brought in DSM by collaborative use (for instance regarding the time intervals) can be addressed by enlarging the binary DSM span of dependencies to ones of the Allen's interval algebra or at least a subset of its dependencies.
\end{abstract}

Keywords: Design Structure Matrix, DSM. Allen's interval algebra. Intervention planning and scheduling. Radiation safety. ALARP/ALARA.

\section{INTRODUCTION - THE CHALLENGES OF THE LHC PROJ ECT}

The CERN Large Hadron Collider (LHC) is a $26.7 \mathrm{~km}$ high-energy proton and ion collider based on several hundred high-field superconducting magnets operating in superfluid helium at $1.9 \mathrm{~K}$. It is the world's most powerful particle accelerator. Straddling the border between France and Switzerland, it has been designed to answer some of the deepest questions of the human kind about the universe: such as the origin of mass, the presence of only matter and not antimatter or the nature of dark matter. It could also provide important new clues about conditions in the very early universe, when the four forces of nature were rolled into one giant super-force.

The idea of such a large particle collider arose back in 1979. After more than a decade of technologies development, the project was approved for construction in 1994. It then took almost 15 years to bring it to operation. In the meantime, a few thousand people were involved in a wide range of activities, from the completion of its R\&D program, to its commissioning with beams in 2008. The technical and managerial expertise of all those who contributed to this project was necessarily a key factor to the success of this unprecedented endeavour.

In the framework of this project, several management and communication means were developed to cope with the requirements of this large-scale project. Shall we recall that the World Wide Web was invented at CERN to facilitate the communication among CERN's scientists who have their home institutes all around the world, who use different operating systems, and who have to share important quantity of data without being TCP/IP experts? CERN has also developed its own PLM system, called EDMS, at the early stages of the LHC project (Bachy 1995). EDMS is an acronym standing for Engineering and Equipment Data Management System (EDMS 2012). As from the late 1990s, this service was available through the Web to the whole LHC project community to upload and download documents and information about the development and construction of the collider, its experiments and the technical infrastructures that house them. Even if the term "Web 2.0" did not exist in the late 1990s_Dale Dougherty used this term for the first time in 2003-, physicists, engineers and technicians involved with this project already experienced a collaborative approach to the Web. To address the planning, scheduling and controlling of the project, CERN also developed a Web tool 
called PPT-EVM aimed at having all the so-called "Project Engineers" (i.e. applied physicists and engineers responsible of a sub-system of the LHC project) participate to the planning, scheduling and controlling of the project and collaborating actively to keep the information it contained up to date. To ease this collaborative process, CERN preferred a deliverable-oriented Earned Value Management approach (Bonnal 2006). By focussing on deliverables, the promoters of this system wanted to avoid the occurrence of the "90\% syndrome" (Ford and Sterman 2003) and to gather the most objective progress information possible. One can say that the objectives of these systems were achieved: the CERN's EDMS is still intensively used by a scientific community, far beyond the CERN boundaries; the CERN's EVM system is still used on projects that are on-going at CERN and is about to be used by large-scale accelerator projects in Russia and in Italy.

As from March 2010, the LHC is in operation (CERN Press Office 2010): proton and heavy ion beams are circulating and colliding into its four major experiments. The LHC is offering to the particle physics community quality data that should lead to the discovery of the existence or inexistence of the Higgs boson, and this before the end of the LHC Run 2012 (CERN Press Office 2012).

The LHC is in operation, beams are circulating, and as a consequence some of its components get activated, i.e. they emit ionizing radiations. The LHC is probably one of the most complex systems human kind has ever built. Like spacecraft, most of its components have been conceived so that maintenance, whether preventive or corrective, is kept to a minimum to limit human interventions on some pieces of equipment that can be activated. Even if a tremendous effort was done in such a direction, some interventions are still required to keep this facility in optimal operating conditions, for physics excellence on the one hand, and for integrity and safety reasons (above all) on the other hand.

After having developed "community tools" for managing effectively the technical side of its facilities (CERN's EDMS), for planning, scheduling and controlling its large-scale projects (CERN's PPTEVM), CERN is now developing two tools: a collaborative Web-based system for planning efficiently and safely interventions in its underground facility and a collaborative Web-based system for maintenance management. This paper aims at presenting some of the key requirements of the so-called IMPACT $^{1}$ system dedicated to intervention planning, and to highlight how DSM can contribute at enhancing the "Web 2.0" dimension of this system. Some of the key requirements of this system are presented in section 2; section 3 gives some insights on how DSM would enhance the "collaborative dimension" of the IMPACT system. Conclusions of this preliminary research work are given in section 4.

\section{INTERVENTION PLANNING AND SCHEDULING REQUIREMENTS}

The foreseen interventions are of several types:

- $\quad$ They can be repair interventions (corrective maintenance) to be performed urgently because an equipment failed or because of an alarm which can occur either during short technical stops organized specifically, or during longer shutdown periods dedicated to maintenance or upgrade of the facilities

- $\quad$ They can also be preventive or predictive maintenance interventions, most often during dedicated shutdown periods

- $\quad$ They can be so-called consolidation interventions that aim at replacing pieces of equipment by newer ones that will enhance the reliability, the availability, the maintainability and the safety (RAMS) of the facilities while the direct operations technical requirements remain the same

- They can be upgrade interventions which consist in the installation and then the commissioning (with or without prior dismounting or dismantling) of new pieces of equipment that are expected to contribute to the achievement of a higher level of performance of the facilities

- $\quad$ They can also be safety and integrity inspections such as radiation surveys or for quality control purposes.

The limits between these activities are not fully tight. For instance, most of the interventions to be performed during the first long shutdown of the LHC, scheduled starting from late 2012 to last 1.5 year are consolidation activities (intended to increase the reliability and the availability of the LHC),

\footnotetext{
${ }^{1}$ Intervention Management Planning and Activity Coordination Tool
} 
but they will also allow to make a step forward in terms of performance: from $4 \mathrm{TeV}+4 \mathrm{TeV}$ collisions in 2012, to $7 \mathrm{TeV}+7 \mathrm{TeV}$ collisions as from 2014 .

From an ontological point of view, these five types of interventions are of two natures: operation-type interventions or project-type interventions.

Most of the consolidation and upgrade interventions are one-of-a-kind activities that are part of projects. They shall be planned according to project planning and scheduling principles. Some repair interventions can also be of this nature, typically if the importance and complexity of the problem is such that their handling by means of the computerized issue management system (CIMS) or of the computerized maintenance management system (CMMS) is not appropriate.

Most of the maintenance interventions, corrective, predictive or preventive, are of operations-type: they are more or less repetitive, and shall be planned and scheduled as per operations management principles: job shop scheduling for instance. Inspections also belong to this nature of interventions.

All these interventions are to be performed in the same facilities, i.e. they all share the same space constraints that are the underground facilities where the accelerators and experiments are installed and the technical buildings where the various services are located. This means that they shall be planned and scheduled within the same intervention planning and scheduling system. To release optimized schedules, this computerized system shall rely on both best practices in matter of operations planning and scheduling and project planning and scheduling.

Moreover, this computerized system shall also consider some specific constraints:

- Safety constraints: hazards and safety risks remain in the facilities even in beam-off mode (Bonnal 2012, §3), which is the case in particular of radiation hazard. For this reason, every job exposing workers to radiations requires a dose plan, and is accepted or rejected following the ALARA or ALARP approach (“As Low As Reasonably Achievable or Possible”). This method consists in an optimized trade-off between the dose received and the means necessary to reduce it further.

- $\quad$ Logistics constraints: CERN's main accelerators and experiment are installed underground (ca. $100 \mathrm{~m}$ below ground level), which makes their access complicated. In addition, since the LHC tunnel is $27 \mathrm{~km}$ long, some equipment can also be located further than $2 \mathrm{~km}$ from any access point, and transportation to installation location can exceed $15 \mathrm{~km}$. For equipment handling reasons, logistics tasks cannot be kept aside and must be part of the intervention schedule.

The combination those constraints leads to the limitation of the number of people present in underground works: in case of an alarm, safety instructions ask them to join a "pressurized safe room" next to the lower station of an elevator prior to evacuate outside.

Logistics is also kept tight to radiation safety, because moving in front of equipment emitting ionizing radiation will give a dose to the worker. The ALARA principle suggests that an optimized route is preferred. Searching this route is not a trivial exercise; it is some kind of travelling salesman problem where a trade-off between the logistic time and the received dose is to be made. To make the problem even more complex: activation levels of equipment vary with time (they will be higher just after the beam is stopped and lower at the end of a shutdown period); some activated equipment is moving; activation levels are difficult to predict and prior in-situ radiation surveys are required.

In summary, the ideal intervention planning and scheduling system shall allow the planning and scheduling of repetitive activities and of one-of-a-kind activities. It shall also allow to take into account important constraints such as safety, radiation safety and logistics constraints. There are no off-the-shelf computerized systems that offer such a service.

\section{A DSM-BASED APPROACH TO ENHANCE COLLABORATION}

In 2010, CERN decided to go ahead in developing a computer system that would ease the planning and scheduling of interventions in underground facilities. As reported in section 1, it is not in the CERN culture to develop centrally managed information systems. And most likely, a few hundred people will interact with this system to submit intervention requests, and then to get intervention authorizations. Several thousands of requests are expected each year; this means that the system shall embed some artificial intelligence means to at least prioritize, pre-plan and pre-schedule interventions prior to their approving and authorization by work superintendents. 
One of the issues of the submission mechanisms to design is how to gather information from the requesters about possible or required interactions and dependencies with other interventions, interventions that have already been submitted to the system, and others. Classically, the critical path method (CPM) and precedence diagramming method (PDM) offer some features using so-called potential constraint types: tasks, i.e. interventions, can be made dependent by finish-start, start-start, finish-finish or start-finish dependencies, with or without lags. Dependent tasks consist in predecessorto-successor couples and CPM and PDM algorithms do not allow transitive closures in the corresponding task network graphs. So-called logistics constraints, i.e. resource constraints can be dealt with by using one of the many resource-constrained project-scheduling algorithms.

While the types of dependency constraints might be appropriate to plan and schedule centrally managed projects, these types of constraints make the implementation of a collaborative project planning and scheduling system difficult: what should be considered if a contributor submits that "task A shall be performed before task B", while another contributor submits the contrary?

Two approaches have been developed to deal with this problem: the so-called design or dependency structure matrix (DSM) on the one hand, the Allen's interval algebra on the other hand.

DSM is a generic matrix-based framework introduced by Steward in the early 1980's (Steward 1981) that provides means to solve out interdependency issues among activities. It was not until the 1990's that the method received strong attention and was applied to the product development process as a powerful management tool (Pimmer and Eppinger 1994, Browning 1998, Eppinger 1997, Smith and Eppinger 1997). It is considered as the most useful tool for analysing highly complicated dependencies, inclusive of feedback and coupled tasks. Dependencies between tasks can be of 3 types as shown on figure 1 .

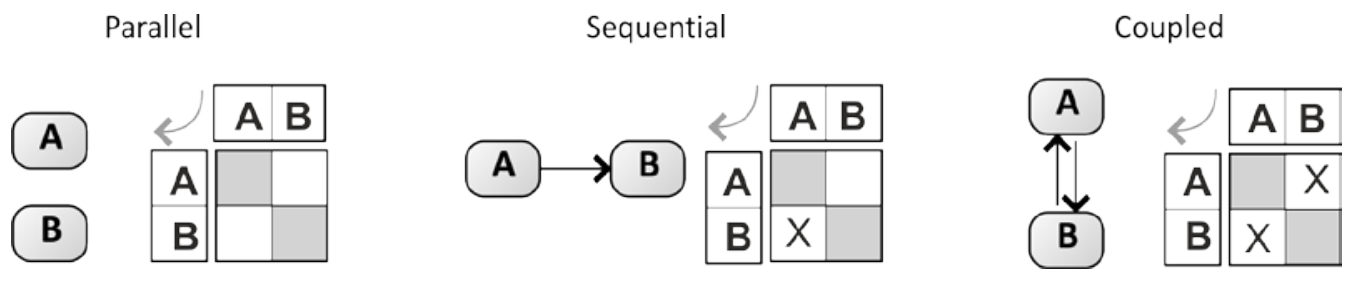

Figure 1. Types of dependencies between tasks of the DSM methodology.

Allen's interval algebra is a calculus for temporal reasoning that was introduced by James F. Allen also in the early 1980's (Allen 1983).

This calculus defines possible relations between time intervals and provides a composition table that can be used as a basis for reasoning about temporal descriptions of events. This algebra proposes 13 possible relations between time intervals (see figure 2) and provides a composition table that can be used as a basis for reasoning about temporal descriptions of events. This algebra can typically be used to evaluate whether a set of dependencies between activities is possible or not. E.g. if someone argues that "task A shall take place before task B" (i.e. A b B), and someone else that "task B shall be overlapped by task A" (i.e. B oi A), then an appropriate use of this combination table will highlight this incoherency. In DSM, this "incoherency" is dealt with by arguing that tasks A and B are coupled, that there is interdependency between these two tasks.

Allen's interval algebra appears to be a powerful tool for dealing with qualitative time intervals; unfortunately it quickly leads to computational tractability problems. Nevertheless, a few attempts have been made to perform project schedule calculations or task networks featuring Allen's event dependencies (Hussain 2000, Smith et al. 2000).

In the framework of intervention planning and scheduling, it is likely that just a subset of Allen's event dependencies are sufficient: for instance A b B and A m B (resp. B bi A and B mi A) can be merged into a unique type of dependency that could feature the finish-start of the critical path method. Figure 2 displays the equivalent DSM notation for each interval dependency. It is clear that the conversion to binary DSM notation sees a loss of information, or necessitates additional entries from the contributor.

For instance, in the cases of "overlap" or "during", the on-going task A may necessitate feedback from the following task B, in which case they are coupled. If not, a step of A might just be needed as a starting event for an independent B which would again be equivalent to the finish-start constraint, and 
would indicate that task A could be further decomposed until a sub-task coupled with B is found or one of the constraint "before" or "meets" is true.

\begin{tabular}{|c|c|c|c|c|}
\hline Relation & Interpretation & Gantt chart-like illustration & \begin{tabular}{|l|l|}
$A B$ \\
$A$ & \\
$B$
\end{tabular} & \begin{tabular}{|l|l|l|}
$A B$ \\
$A$ & $x$ \\
$B$ & $X$ \\
\end{tabular} \\
\hline $\begin{array}{l}A \text { b B } \\
B \text { bi } A\end{array}$ & $\begin{array}{l}\text { A takes place before B } \\
\text { B takes place after A }\end{array}$ & $\begin{array}{ll} \\
\end{array}$ & $\mathbf{X}$ & \\
\hline $\begin{array}{l}\text { A m B } \\
\text { B mi A }\end{array}$ & $\begin{array}{l}A \text { meets } B \\
B \text { is met by } A\end{array}$ & $\begin{array}{ll}A \\
\end{array}$ & $X$ & \\
\hline $\begin{array}{l}A \circ B \\
B \text { oi } A\end{array}$ & $\begin{array}{l}\text { A overlaps B } \\
B \text { is overlapped by A }\end{array}$ & $\begin{array}{l}\mathbf{A} \\
\mathbf{B}\end{array}$ & $X$ & $x$ \\
\hline $\begin{array}{l}A s \mathrm{~B} \\
B \operatorname{si} A\end{array}$ & $\begin{array}{l}\text { A starts B } \\
\text { B is started by A }\end{array}$ & \begin{tabular}{|c|}
$\mathbf{A}$ \\
$\mathbf{B}$ \\
\end{tabular} & & $X$ \\
\hline $\begin{array}{l}A d B \\
B d i A\end{array}$ & $\begin{array}{l}A \text { is during } B \\
B \text { contains } A\end{array}$ & \begin{tabular}{|c|} 
A \\
B \\
\end{tabular} & $X$ & $X$ \\
\hline $\begin{array}{l}A f B \\
B f i A\end{array}$ & $\begin{array}{l}\text { A finishes } B \\
B \text { is finished by } A\end{array}$ & \begin{tabular}{|c|} 
A \\
B \\
\end{tabular} & & X \\
\hline$A=B$ & A equals $B$ & \begin{tabular}{|l|} 
A \\
B \\
\end{tabular} & & $\mathbf{X}$ \\
\hline
\end{tabular}

Figure 2. Allen's interval algebra dependency types between events with DSM transcription.

\section{CONCLUSION}

Most of the applications of DSM are aimed at dealing with the complexity of the early stage of development projects, i.e. the system-level design and detailed design phases as per Ulrich and Eppinger (2003) for instance. DSM can also provide means to solve out planning and scheduling problems during integration and commissioning phases of development projects. Some works also show that this methodology can be quite useful for planning and scheduling maintenance activities (e.g. Kiss et al. 2011). We believe that the DSM approach can be particularly well suited in the framework of a collaborative intervention planning and scheduling system for collecting dependencies between requested interventions. But gathering dependency information in a binary form-no dependency or finish-start dependency (coupled dependencies derive from opposite finish-start submissions) - is probably not sufficient, it is certainly wise to consider a larger span of dependencies and Allen's interval algebra offers such a means.

"Web 2.0" systems have many promoters. They also raise criticisms: these systems are prone to chaos. Those systems that have moderators are more structured; information is easier to retrieve. The tradeoff then becomes: how much moderation effort to allocate to keep a system sustainable while keeping the "Web 2.0" flexibility? We believe that the optimization of this trade-off relies on the upfront design of a system that embeds an important amount of structured information; the combination of DSM and of Allen's interval algebra dependencies should contribute to the design of such a system.

\section{ACKNOWLEDGEMENTS}

This research project has been supported by a Marie Curie Actions Initial Training Network Fellowship of the European Community's Seventh Framework Programme (FP7) under contract number (PITN-GA-2010-264336-PURESAFE).

\section{REFERENCES}

Allen, F. A. (1983) Maintaining knowledge about temporal intervals. In: Communications of the ACM. 26 November 1983. ACM Press. 832-843. 
Bachy, G., Hameri, A.-P. (1995) What has to be implemented at the early stage of a construction project. Int. J. Project Manage. 15 (4) 211-218.

Bonnal, P., De Jonghe, J., Ferguson, J. (2006) A deliverable-oriented EVM system suited to a largescale project. Project Manage. J. 37 (1) 67-80.

Bonnal, P. editor (2012) Working on the CERN site. https://edms.cern.ch/document/1155899/2.0

Browning, T.R. (1998) Use of dependency structure matrices for product development cycle time reduction. In: Prasad, B. editor. Proceedings of the Fifth ISPE International Conference on Concurrent Engineering: Research and Applications, Tokyo, Japan, 15-17 July 1998.

CERN EDMS Service website (2011) https://espace.cern.ch/edms-services/services/default.aspx [22 April 2012].

CERN Press Office (2010) LHC research programme gets underway http://cern.ch/press/PressReleases/Releases2010/PR07.10E.html [22 April 2012].

CERN Press Office (2012) LHC physics data taking gets underway at new record collision energy of $8 \mathrm{TeV}$ http://cern.ch/press/PressReleases/Releases2012/PR10.12E.html [22 April 2012].

Eppinger, S.D. (1997) A planning method for integration of large-scale engineering systems. In: Riitahuhta, A. editor. Proceedings of the International Conference on Engineering Design (ICED-97), Tampere University of Technology, Finland, 19-21 August 1997. 199-204.

Ford, D.N., Sterman, J.D. (2003) Overcoming the 90\% Syndrome: Iteration management in Concurrent Development Projects. Concurrent Engineering 11 177-186.

Hussain, S. (2000) Project management using temporal logic programming. In: 2000 IEEE Int. Conference on Systems, Man, and Cybernetics. Nashville, TN. Oct. 2000. 2144-2149.

Kiss, J. et al. (2011) Matrix-Based Methods for Planning and Scheduling Maintenance Projects. In: Proceedings of the 13th International DSM Conference. MIT, Cambridge, MA, USA. October 2011. 421-426.

Pimmer, T.U., Eppinger, S.D. (1994) Integration analysis of product decompositions. ASME Conference on Design Theory and Methodology 68 343-351.

Smith, D. E. ; Frank, J. ; Jónsson, J. D. (2000) Bridging the gap between planning and scheduling. Knowledge Eng. Rev. 15 (1) 47-83.

Smith, R.P, Eppinger, S.D. (1997) Identifying controlling features of engineering design iteration. Management Science 43 (3) 276-93.

Steward, D. (1981) The design structure system: a method for managing the design of complex systems. IEEE Transactions on Engineering Management 28 (3) 71-74.

Ulrich, K.T., Eppinger, S.D. (2003) Product design and development. $3^{\text {rd }}$ ed. New-York: McGraw Hill Publishers. 366 p.

\section{Contacts:}

Mathieu Baudin, CERN, Office of the Director for Accelerators and Technology

(Early-stage researcher with the PURESAFE Marie Curie Actions Initial Training Network)

Mailbox M22100, CH-1211 Geneva, Switzerland, +41 2276622 34, mathieu.baudin@cern.ch

Pierre Bonnal, CERN, Office of the Director for Accelerators and Technology

(CERN's scientist-in-charge for the PURESAFE Marie Curie Actions Initial Training Network)

Mailbox M22210, CH-1211 Geneva, Switzerland, +41 2276757 10, pierre.bonnal@cern.ch

Jurgen De Jonghe, CERN, General Infrastructure Services Dept., Advanced Information Systems Group

Mailbox C04800, CH-1211 Geneva, Switzerland, +41 2276781 97, jurgen.de.jonghe@cern.ch 